\title{
The Life Cycle of Baroclinic Eddies in a Storm Track Environment
}

\author{
ISIDORO ORLANSKI AND BRIAN GROSS \\ Geophysical Fluid Dynamics Laboratory, Princeton, New Jersey
}

(Manuscript received 15 April 1999, in final form 1 March 2000)

\begin{abstract}
The life cycle of baroclinic eddies in a controlled storm track environment has been examined by means of long model integrations on a hemisphere. A time-lagged regression that captures disturbances with large meridional velocities has been applied to the meteorological variables. This regressed solution is used to describe the life cycle of the baroclinic eddies. The eddies grow as expected by strong poleward heat fluxes at low levels in regions of strong surface baroclinicity at the entrance of the storm track, in a manner similar to that of Charney modes. As the eddies evolve into a nonlinear regime, they grow deeper by fluxing energy upward, and the characteristic westward tilt exhibited in the vorticity vanishes by rotating into a meridional tilt, in which the lower-level cyclonic vorticity center moves poleward and the upper-level center moves equatorward.

This rather classical picture of baroclinic evolution is radically modified by the simultaneous development of an upper-level eddy downstream of the principal eddy. The results suggest that this eddy is an integral part of a self-sustained system here named as a couplet, such that the upstream principal eddy in its evolution fluxes energy to the upper-level downstream eddy, whereas at lower levels the principal eddy receives energy fluxes from its downstream companion but grows primarily from baroclinic sources. This structure is critically dependent on the strong zonal variations in baroclinicity encountered within the storm track environment.

A second important result revealed by this analysis is the fact that the low-level vorticity centers that migrate poleward tend to follow isotachs that closely correspond to the phase speed of the eddies. It is suggested that the maximum westward momentum that the eddies deposit at lower levels corresponds to the phase velocity, a quantity that can be estimated just from the upstream conditions. The intensity and direction of propagation of these waves will determine the overall structure of the storm track.
\end{abstract}

\section{Introduction}

Storm tracks are defined as the regions where eddy activity is the strongest. They are characterized by zonal asymmetry-with a distinct entrance where surface baroclinicity is strongest and eddies are generated; a large middle region where the baroclinicity is weaker but baroclinic eddy activity is strong and the amount of eddy energy is largest; and a termination region where eddies break, there is very low surface baroclinicity, and there is virtually no eddy generation. A new picture is now emerging of the role of highly transient eddies in storm track environments, based on studies with observations and GCMs. These studies suggest that baroclinic eddies play a very important role in developing and maintaining long-lived blocking structures (Illari and Marshall 1983; Nakamura et al. 1997) and are even able to shape the quasi-stationary circulation of the storm tracks (Hoskins et al. 1983; Orlanski 1998, hereafter O98). The forcing exerted by the high-frequency eddies on flow

Corresponding author address: Dr. Isidoro Orlanski, Geophysical Fluid Dynamics Laboratory, Princeton, NJ 08542.

E-mail: io@gfdl.gov patterns of longer timescale is mainly due to the way in which those eddies tend to decay or break in particular regions of the storm track. The high-frequency eddy kinetic energy and the forcing of the barotropic vorticity in the Pacific storm track are shown in Fig. 1a. The baroclinic eddies exert a cyclonic forcing at the western poleward side of the storm track and anticyclonic forcing at the eastern equatorward side of the storm track. This forcing is consistent with the trough-ridge system that characterizes the winter conditions at the entrance and termination of the storm track.

These diagnostics indicate that these different regions might support qualitative differences in the development and decay of eddies. Most of our understanding of eddy life cycles has emerged from investigations examining normal modes on zonally symmetric jets. Nonlinear life cycle experiments using the fastest growing linear baroclinic normal modes on a basic zonal flow exhibit many of the characteristics of observed midlatitude storms (e.g., Simmons and Hoskins 1978, 1980; Balasubramanian and Garner 1997a,b; among others). Observational studies have concluded that the basic structure of midlatitude baroclinic eddies are indeed very similar to those of nonlinear baroclinic normal modes (Lim and Wallace 1991; Chang 1993). 


\section{Observed}

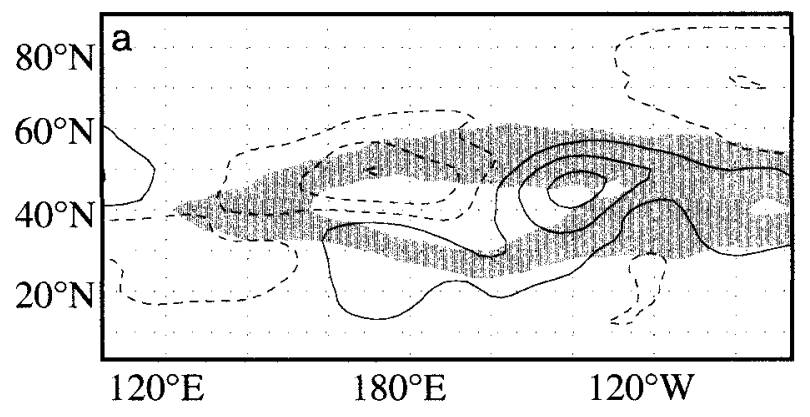

Simulated

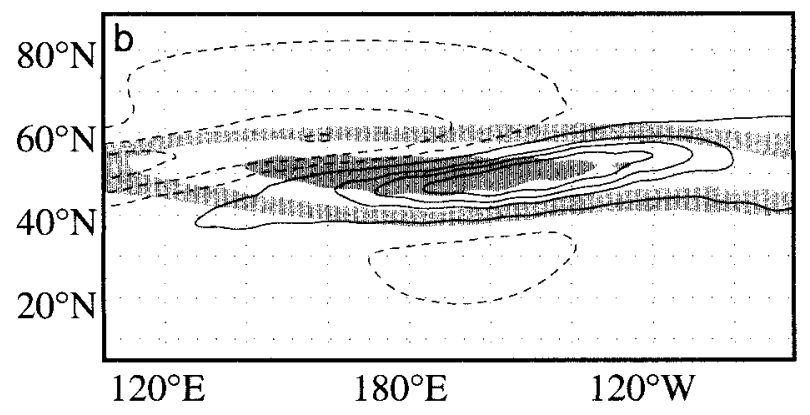

FIG. 1. Vertically averaged eddy kinetic energy (color shading every $30 \mathrm{~m}^{2} \mathrm{~s}^{-2}$ ) and streamfunction tendency (contoured every $10 \mathrm{~m}^{2}$ $\mathrm{s}^{-2}$; negative contours dashed) in the (a) observations [Dec mean from 1980 to 1990, reproduced from Orlanski (1998)] and (b) numerically simulated storm track. Both plots represent 200-day averages. The streamfunction tendency is derived from the vertically averaged high-frequency forcing, and high-frequency quantities were derived using a 6-day high-pass filter, as described in Orlanski (1998).

However, considerable differences exist in the evolution of the observed eddies in different regions of the storm track. At the storm track entrance, eddy growth may resemble the initial normal-mode evolution. However, Chang (1993) has shown with a regression analysis on the observed data over the Pacific storm track that eddies in the middle of the storm track can grow and decay by fluxing energy downstream as suggested in previous studies (Simmons and Hoskins 1979; Chang and Orlanski 1993). Moreover, the abrupt zonalization due to barotropic decay characteristic of baroclinic normal modes has been questioned by Simmons and Hoskins (1978) as quite unrealistic and uncharacteristic of storm track termination.

It is assumed that the zonal asymmetries of the storm track substantially modify the characteristics of baroclinic growth. Normal modes grow by pure baroclinic process and decay by barotropic momentum fluxes, whereas storm track eddies develop by a combination of baroclinic processes and upstream energy fluxes and could decay by a number of different processes, such as momentum fluxes, wave breaking, and downstream energy fluxing. It can be assumed then that neither the growth nor decay stages of these eddies resembles normal-mode evolution. The focus of this paper will be an examination of the characteristics of the growth and decay of baroclinic eddies in a controlled storm track environment.

\section{Experimental setup \\ a. Atmospheric model}

The results presented here were achieved by means of numerical model simulations using the anelastic hydrostatic version of the Geophysical Fluid Dynamics Laboratory ZETA model (Orlanski and Gross 1994). This model employs a terrain-following vertical coordinate based on physical height that provides higher vertical resolution near the ground. The simulations were performed in a channel consisting of an 18-kmdeep zonally periodic global latitude band bounded by impermeable walls at $0^{\circ}$ and $85^{\circ} \mathrm{N}$. The horizontal resolution is about $75 \mathrm{~km}$, and the vertical resolution ranges from $320 \mathrm{~m}$ near the surface to about $1 \mathrm{~km}$ near the rigid lid. Physical processes include Laplacian horizontal smoothing, surface friction that derives from a surface stress based on a simple drag law and a first-order closure scheme above the surface (Haltiner and Williams 1980, chapter 8), and vertical mixing using a diffusion coefficient that depends on the local Richardson number. The initial conditions for the simulations consist of the zonally symmetric state, shown in Fig. 2, which is characterized by a baroclinic jet, its associated meridional potential temperature gradient, and nearly uniform potential vorticity below the tropopause. The shape of this jet is similar to that used by Simmons and Hoskins (1979). Here, however, the jet profile is given by $U(\varphi, z)=\left(U_{0}+\Lambda z\right)\{\sin [\pi \sin (\varphi)]\}$, which is more meridionally confined than the Simmons and Hoskins profile in order to mimic the jet at the entrance of a storm track. The maximum speed of the jet is about 70 $\mathrm{m} \mathrm{s}^{-1}$, which is faster than typically observed zonal mean flows, but it corresponds well with the speed of the zonal jet at the entrance of observed storm tracks. The storm track simulations were run for 420 model days, the last 200 of which are used to construct the regressions and time means presented here.

\section{b. Maintenance of the basic state}

Near the upstream (western) boundary, placed in this presentation at longitude $0^{\circ}$, the basic state is maintained by Newtonian damping within a sponge region that is approximately $11^{\circ}$ wide and confined between $20^{\circ}$ and $70^{\circ} \mathrm{N}$. This appears sufficient to maintain a region of baroclinicity in which eddies can develop. Periodicity returns the flow to the basic state at the eastern end of the channel, well beyond the termination of the storm track. Additionally, within this sponge region the initial state is continuously perturbed by a $1 \mathrm{~m} \mathrm{~s}^{-1}$ meridional 


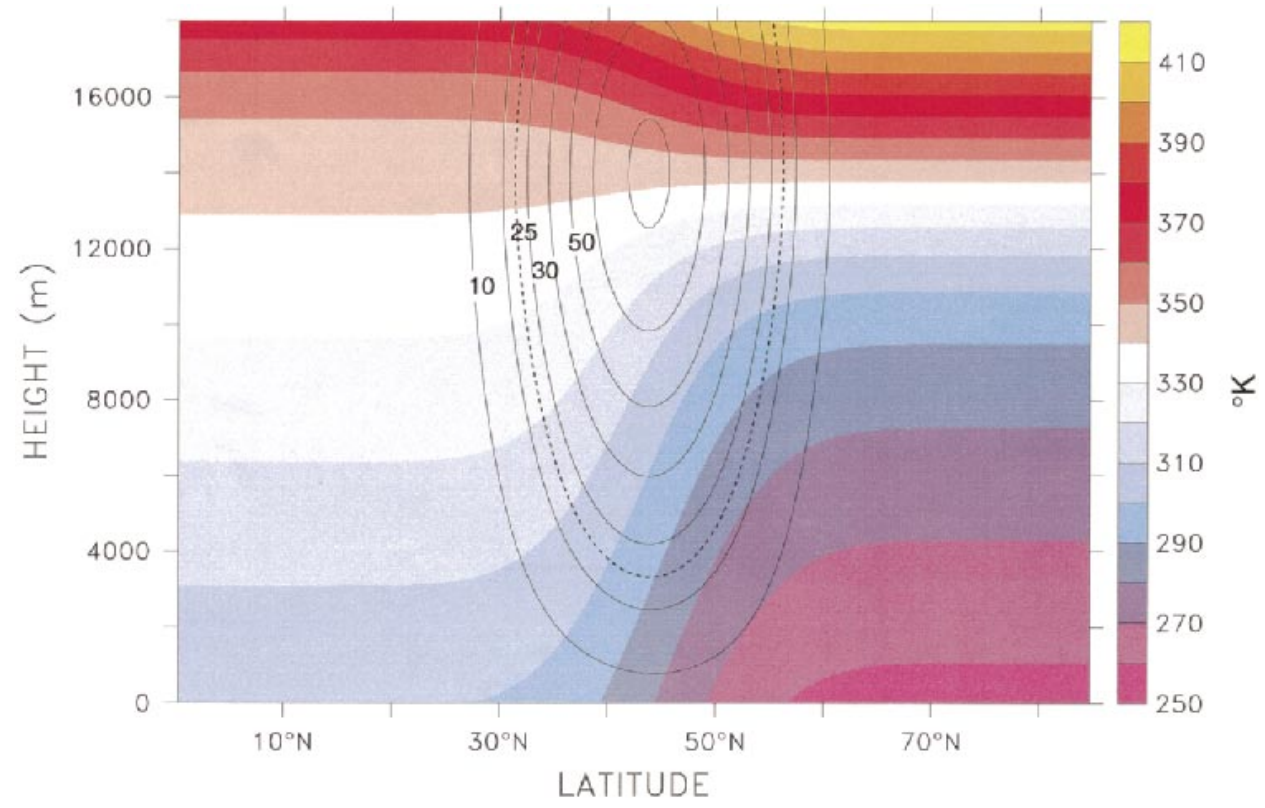

FIG. 2. Basic-state zonal wind (contoured every $10 \mathrm{~m} \mathrm{~s}^{-1}$; zero contour heavy, negative values dashed) and potential temperature (color shading in $\mathrm{K}$ ). The heavy dashed contour corresponds to a time-mean zonal wind of $U_{0} \approx 5 \mathrm{~m} \mathrm{~s}^{-1}$, the approximate phase speed of the eddies in the storm track.

velocity variation at a longitude just downstream of the western boundary and extending throughout the depth of the domain. The velocity perturbation changes sinusoidally in time with a period of $10^{5} \mathrm{~s}$. Advection of this perturbation out of the sponge region by the mean flow provides a perturbation wavelength of about 3800 $\mathrm{km}$. These perturbations act as seeds for baroclinic development at the entrance of the storm track.

Traditionally, the basic state is maintained by zonally symmetric interior forcing. This approach is not adopted here so that the interior solution can freely respond to the eddy forcing. Instead, the sponge maintains a flow at the upstream boundary similar to the subtropical jet that is commonly observed at the entrance of the storm track. As a consequence, these experiments do not represent the true large-scale variability in which storm tracks are imbedded, but rather they can be used to examine the mutual dynamical forcing between baroclinic eddies and a zonal flow.

\section{Life cycle as inferred from regression analysis}

Chang and Orlanski (1993) previously attempted to clarify the life cycle of baroclinic eddies in storm track simulations. One of their main conclusions was that, although eddies grow mainly by baroclinic processes at the entrance of the storm track, as normal-mode studies suggest, upper-level eddies near the middle of the storm track can grow as a result of energy fluxes from upstream as well as from the surface baroclinicity. These results seem to explain why the eddy kinetic energy maximum is downstream of the maximum in baroclinicity.
One of the shortcomings of these earlier model studies is that the simulated eddies tended to disperse energy nearly uniformly in the zonal direction, without producing a termination of the storm track itself, in contradiction to observed behavior (O98). It is believed that this failure was due to the Cartesian geometry of the model. Recent normal-mode studies (Whitaker and Snyder 1995; Balasubramanian and Garner 1997a) have shown dissimilar evolution of baroclinic normal modes in spherical and Cartesian systems; the large-scale eddies tended to break anticyclonically in the spherical case and cyclonically in the Cartesian geometry. Since the characteristic anticyclonic circulation at the termination of the storm track was never achieved in the Cartesian geometry, anticyclonic wave breaking may be paramount in terminating the storm track.

Here, a control simulation of a storm track was produced by running a hemispheric model in spherical coordinates for 400 days and prescribing the meridional structure of a jet in a confined longitudinal band representing the entrance of the storm track. The eddy statistics for one of the experiments is shown in Fig. 1b. A remarkable similarity of the idealized storm tracks with the observed one can be noticed. Cyclonic and anticyclonic breaking tend to produce a trough-ridge system characteristic of observed storm tracks (O98).

In this presentation, the life cycle of these eddies will be systematically analyzed by means of regression analyses. Wallace et al. (1988) and Chang (1993), among others, have established that an objective composite of eddies in the storm track environment can be constructed from a regression analysis. The regression 


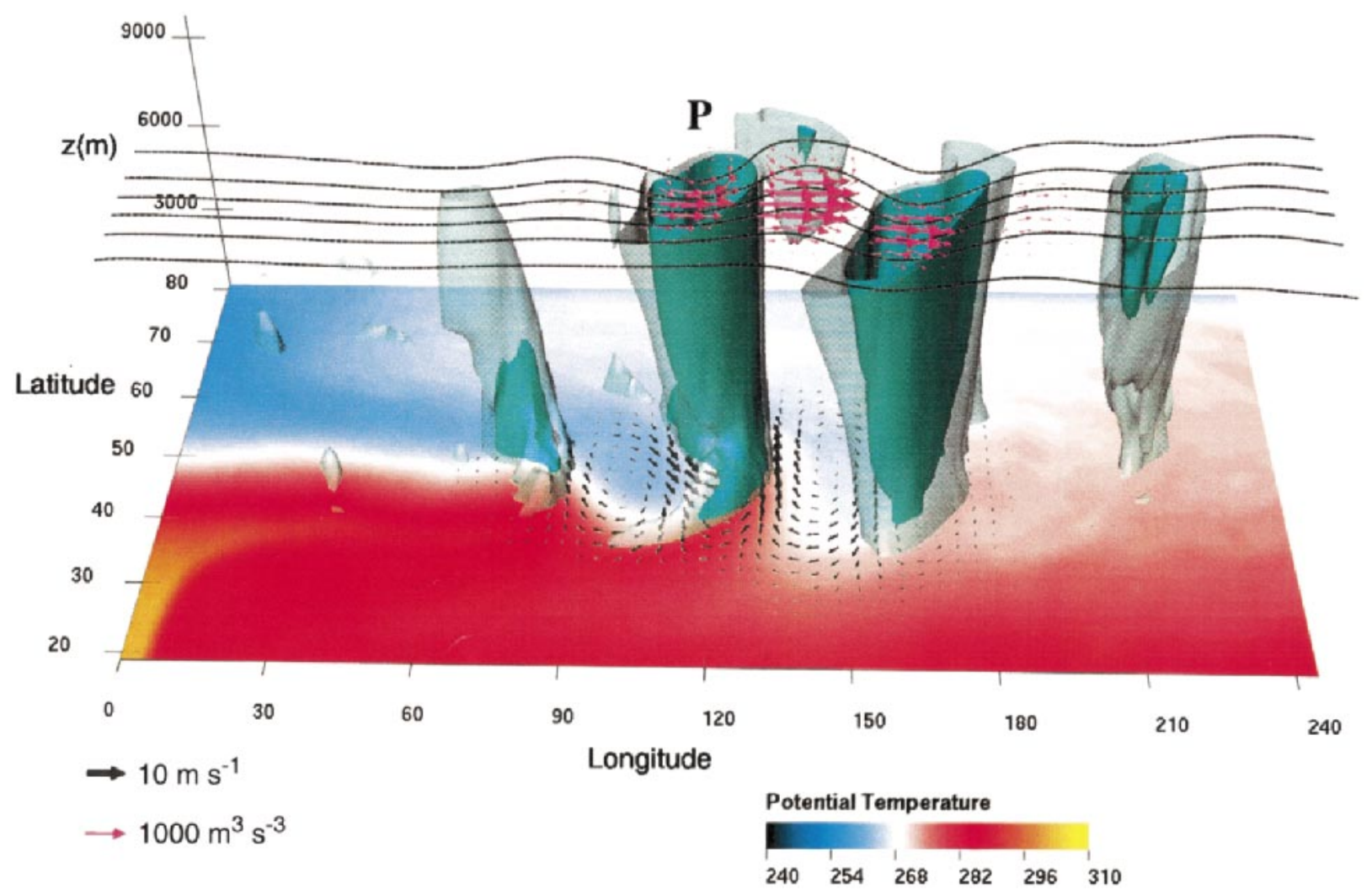

FIG. 3. Regression with zero time lag of the cyclonic relative vorticity (light blue $3 \times 10^{-6} \mathrm{~s}^{-1}$ and dark blue $7 \times 10^{-6} \mathrm{~s}^{-1}$ isosurfaces), surface potential temperature (color shading in K), surface wind (black vectors), ageostrophic geopotential fluxes at $10 \mathrm{~km}$ (magenta vectors), and geopotential at $10 \mathrm{~km}$ (contours every $2190 \mathrm{~m}^{2} \mathrm{~s}^{-2}$ ). The largest surface wind vector corresponds to about $8 \mathrm{~m} \mathrm{~s}^{-1}$, and the largest geopotential flux vector corresponds to about $1500 \mathrm{~m}^{3} \mathrm{~s}^{-3}$. "P" denotes the principal eddy.

method used by Lim and Wallace (1991) and Chang (1993) will also be used here. The regression coefficient $X_{R}(x, y, z, \tau)$ is given by

$$
X_{R}(x, y, z, \tau)=\frac{1}{N} \sum_{t} X(x, y, z, t) T_{E}(t-\tau),
$$

where the regressed variable $X$ represents the deviations from the time mean and $T_{E}$ is the normalized reference time series. In this definition the regression coefficient $X_{R}$ carries the units of the regressed variable. Different meteorological variables have been used to construct the time series for the test function $T_{E}$. Chang (1993) found it convenient to use the meridional velocity at a particular location for $T_{E}$. His argument was that because the time series he used were not filtered in time, the meridional velocity can better capture the high-frequency eddies. However, when both phases of the meridional velocity are included in the test function, the cyclonic and anticyclonic phases of the wave are averaged together, destroying the positional and structural differences between these two phases. In order to preserve these differences, the following test function was chosen because it can discriminate between wave phases:

$$
T_{E}(t)=\frac{1}{\operatorname{var}(V)} \begin{cases}V^{2}\left(x_{0}, y_{0}, z_{0}, t\right) & \text { if } V>0 \\ 0 & \text { if } V<0 .\end{cases}
$$

Additionally, this test function selects the eddies of largest amplitude, which tend to produce frontal occlusion and temperature roll-up at lower levels. Choosing the negative phase in (2.2) gives generally similar regressed fields, but with the corresponding change in sign and characteristic shape.

A composite of cyclone evolution within a storm track is depicted in Fig. 3, in which isosurfaces of cyclonic relative vorticity, the surface potential temperature and wind vectors, and upper-level energy fluxes and geopotential are displayed. All of these quantities are derived from the regressed solution. The location of the test function was selected by identifying the area of intense wave breaking closest to the entrance of the storm track, at a height of $4 \mathrm{~km}$. This level was chosen so that the characteristics of wave breaking could be captured reasonably well at all levels. The test function used in the following discussion is $V^{2}\left(x_{0}=134^{\circ} \mathrm{E}, z_{0}\right.$ $=4 \mathrm{~km}, t$ ), averaged over a 15 -band centered at $52.5^{\circ} \mathrm{N}$.

Total fields in Fig. 3 (surface potential temperature and upper-level geopotential) are constructed by adding 
the normalized regressed fields to their respective time means. The normalization is achieved with a scaling factor, chosen to reflect the structure in a typical largeamplitude wave (examples will be shown below), which at zero lag is characterized by a surface potential temperature field that is on the verge of overturning meridionally. ${ }^{1}$

At the entrance of the storm track, a growing eddy is observed within which baroclinicity generates the low-level vorticity. The vorticity surface is tilted westward, a well-known feature of unstable baroclinic eddies. As this system matures, the upper and lower relative vorticity centers undergo a rotation, during which the low-level cyclone center moves poleward during its roll-up, whereas the upper-level center moves equatorward. These features are apparent in the principal eddy, centered at $120^{\circ}$ longitude and denoted by "P," which shows features typical of a mature eddy, including the cold frontal structure behind warm (red) air advected from the south, and the associated cyclonic surface circulation. The open cone shape of the vorticity isosurface at $10 \mathrm{~km}$ indicates that the upper-level vorticity has considerable meridional structure, with its center south of the surface center. As the low-level cyclonic circulation rolls up the potential temperature field to produce the gradient reversal in the poleward flank, energy fluxes (magenta vectors) predominate at upper levels, consistent with the downstream development mechanism. These energy fluxes tend to be quite dispersive, that is, with a considerable equatorward component, which produces an upper-level disturbance that tends to move equatorward, as in the third vorticity center (referred to hereafter as the downstream eddy). As will be discussed below, the principal eddy and the downstream eddy can be considered a distinctly nonmodal couplet, where the principal eddy is maintained by baroclinicity and the downstream eddy by geopotential fluxes from the principal eddy. The anticyclonic shear on the equatorward side of the jet shreds the downstream eddy, which breaks anticyclonically. Inspecting different time lags in the regression reveals that the downstream system will evolve without further regeneration because at this point in the storm track surface baroclinicity has been severely depleted. In these experiments this is in part an artifact of forcing baroclinicity only at the upstream boundary, but these conditions are clearly evident in observations (e.g., Hoskins and Valdes 1990; Orlanski 1998) that show considerably less baroclinicity in the eastern half of the oceans than in the western half. In the following sections each of the important features shown in Fig. 3 will be more fully discussed.

It is satisfying that the regressed solution shown in

\footnotetext{
${ }^{1}$ Since only one phase is used in (2.2), the amplitude of the regressed field is half of that had both phases been used. A scaling factor of 3 was chosen so that the regressed fields have an amplitude corresponding to 1.5-2 standard deviations.
}

Fig. 3 captures most of the features of observed baroclinic development that have been collected from many studies on the evolution of cyclones. The eddies within the storm track start out as classic shallow Charney modes, in which vertical fluxing of energy transforms it into something resembling an Eady mode. As the horizontal scale grows, upper-level energy fluxes produce downstream disturbances at upper levels that are equivalent barotropic and that eventually elongate and become shredded by the jet. The very weak surface baroclinicity prevents the redevelopment of these upperlevel disturbances into full-fledged baroclinic waves. These features nicely agree with Hoskins's (1983) qualitative description of the stages of cyclone evolution, but the experiments described here will clarify the relevant mechanisms in these stages: linear growth; followed by nonlinear cessation of growth leading to Rossby wave radiation upward and then equatorward; and finally an absorption process akin to critical layer absorption on the tropical flank of then jet, which in our case is represented by eddy elongation and shredding.

\section{Evolution of the baroclinic eddies}

\section{a. The principal eddy}

The principal eddy exhibits the characteristic structure of baroclinic development, as shown in Fig. 4. A positive vorticity maximum located west of the maximum warm anomaly provides large poleward advection of the positive temperature anomaly coupled with strong equatorward advection of the cold-temperature anomaly behind the front (Fig. 4a). The heavy weighting of very strong systems by the test function used in this regression produces reversed temperature gradients in the eddies at zero lag. In fact, the total potential temperature gradient is reversed as well, as shown in Fig. 3. Further evidence of baroclinic development is provided by the large meridional heat fluxes depicted in Figs. 4b,c. Here, very strong positive meridional heat fluxes at the surface occur ahead of the cold front, while sinking cold air in the trailing system provides a weaker positive heat flux. At upper levels, the vorticity center associated with the principal eddy also has poleward heat fluxes to the east due to warm air ascending ahead of this eddy. Cold-air descent within the eastern half of the downstream ridge also provides positive heat fluxes. The heat fluxes associated with this mature eddy are already smaller than at earlier times, when the eddy was rapidly developing. The extrema in vorticity and heat flux at the surface (Fig. 4b) are oriented in a northwest direction, while those at $10 \mathrm{~km}$ (Fig. 4c) are oriented in a northeast direction. Assuming that the phase velocity vector is perpendicular to this orientation suggests that at low levels the system propagates to the northeast, while at upper levels they will move to the southeast.

One of the interesting features revealed by this regression is the fact that the westward tilt of the principal 

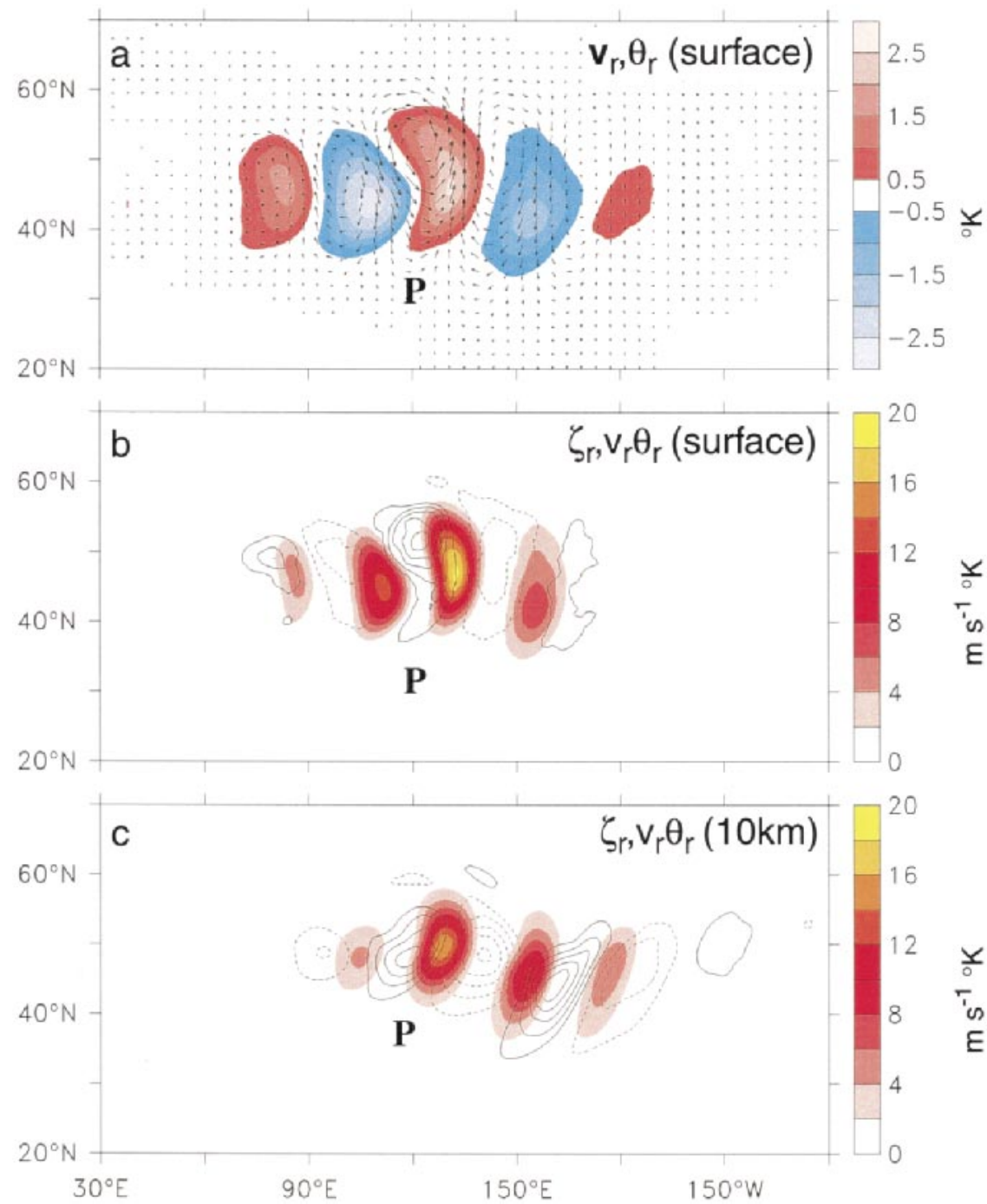

FIG. 4. (a) The regressed surface potential temperature anomaly (color shading in K) and wind at 0 lag. The largest surface wind vector corresponds to about $8 \mathrm{~m} \mathrm{~s}^{-1}$. Only a portion of the model domain is shown. (b) The regressed surface meridional heat flux (color shading in $\mathrm{m} \mathrm{s}^{-1}$ $\mathrm{K}$ ) and relative vorticity (contoured every $6 \times 10^{-6} \mathrm{~s}^{-1}$; negative contours dashed). (c) The same as (b) but at $10 \mathrm{~km}$. "P" denotes the principal eddy.

eddy required for baroclinic intensification rotates into a meridional tilt as the eddy grows. This rotation may in fact comprise a fundamentally nonlinear aspect of baroclinic instability. These features are clearly seen in a two-dimensional depiction of vortex rotation (Fig. 5). Also apparent in this figure is the distinct poleward propagation of the surface cyclone and the equatorward movement and meridional extension of the eddy at upper levels.

One way to interpret the movement of surface lows northward and upper-level highs southward is by means of a simple quasigeostrophic argument (Hoskins 1976; Hoskins and West 1979). The depletion of baroclinicity will by geostrophic adjustment produce surface westerlies and upper-level easterlies. This modification of the zonal wind is consistent with enhanced low pressure to the north at the surface and enhanced low pressure to the south at upper levels. From the eddy's perspective, this displacement can be viewed as a rotation of the westerward tilt at the initial stages of eddy development into a meridional tilt in the mature phase of the system.

This description of the rotation of the westward tilt into a meridional tilt can be interpreted using an inspection of the vorticity budget, which clarifies the physical mechanisms behind the movement and intensification of the principal vortex. At the surface, advection of regressed vorticity by the mean flow simply moves the eddy eastward, but at a speed less than the phase speed of the wave (here empirically determined to be $U_{0} \approx 25 \mathrm{~m} \mathrm{~s}^{-1}$ ). However, as shown in Fig. $4 \mathrm{~b}$, 


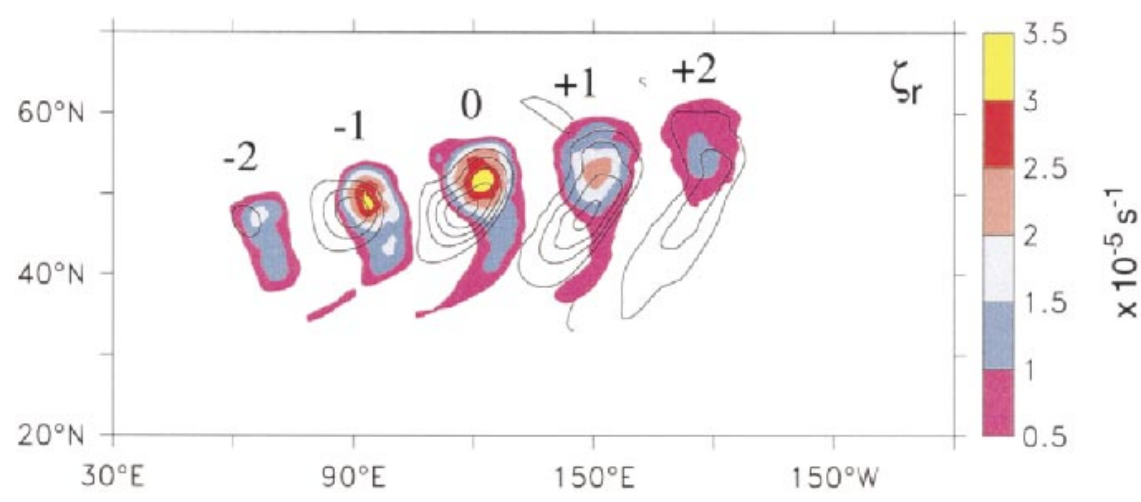

FIG. 5. The cyclonic relative vorticity at the surface (color shading in $10^{-5} \mathrm{~s}^{-1}$ ) and at $10 \mathrm{~km}$ (contoured every $0.5 \times 10^{-5} \mathrm{~s}^{-1}$ ) at the lags (in days) indicated by the labels.

the heat flux convergence and the associated increase in vertical stretching has a maximum to the northeast of the principal eddy, and the fluxes themselves are oriented in a poleward direction. Nonlinear vorticity fluxes, composed of nonlinear advection and self-induced stretching, also enhance cyclonic vorticity to the northeast. These terms combine to steer the surface eddy in a northeastern direction, as shown in Fig. 6a, at a speed corresponding to the phase speed of the wave.

At upper levels, advection by the mean flow moves the eddy eastward as at the surface, but at speeds greater than the phase speed of the wave. The flux of mean absolute vorticity by the regressed velocity compensates the mean advection, so that the upper-level wave prop- agates at the phase speed. Nonlinear advection provides weak positive (cyclonic) tendencies northeast of the eddy and negative (anticyclonic) tendencies to the southeast. This will promote the elongation of the upperlevel eddy into a more north-south orientation. The total tendency is shown in Fig. 6 b.

\section{b. The development of the couplet system}

One of the most important features seen in the regressed upper-level vorticity (Fig. 4c) is that the strongest vorticity is not associated with the principal eddy but rather is associated with the cyclonic eddy downstream of the principal eddy. Figure 3 shows strong

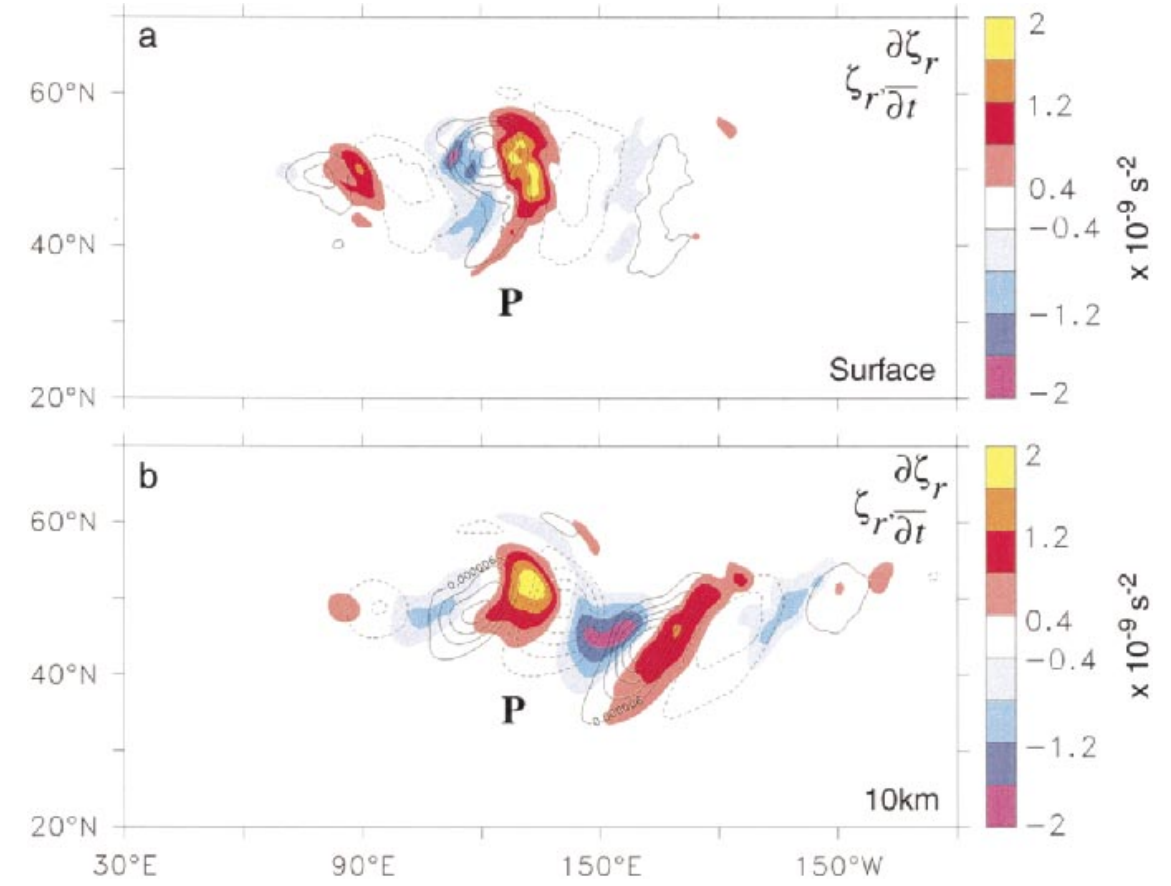

FIG. 6. (a) Total relative vorticity tendency (color shading in $10^{-9} \mathrm{~s}^{-2}$ ) and relative vorticity (contoured every $6 \times 10^{-6} \mathrm{~s}^{-1}$ ) at (a) the surface and (b) $10 \mathrm{~km}$. "P" denotes the principal eddy. 


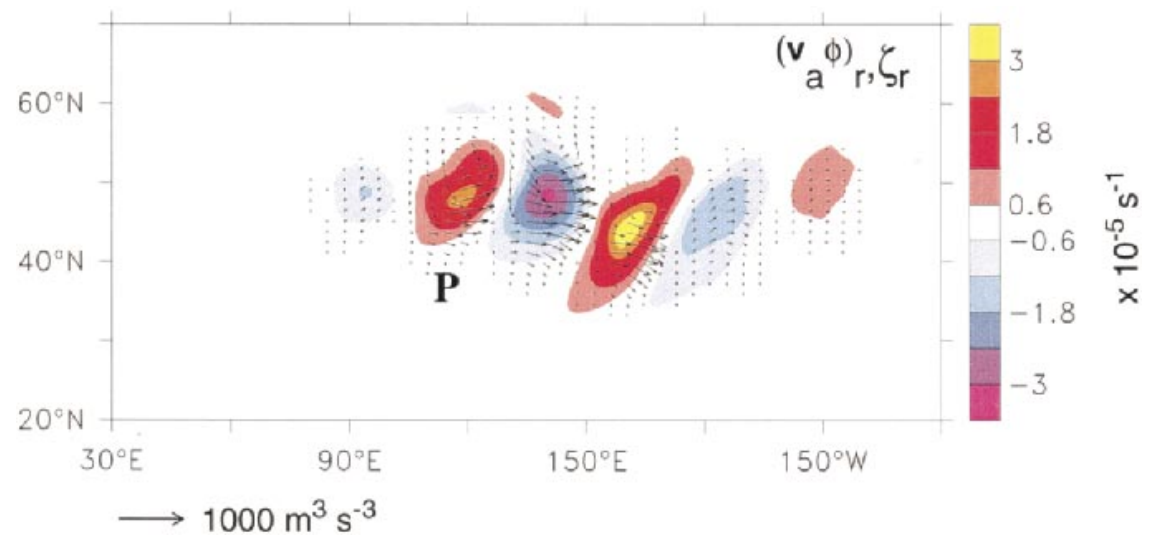

FIG. 7. Ageostrophic geopotential flux vectors and the relative vorticity (color shading in $10^{-5}$ $\mathrm{s}^{-1}$ ) evaluated at $10 \mathrm{~km}$. The longest flux vector corresponds to about $1000 \mathrm{~m}^{3} \mathrm{~s}^{-3}$. "P" denotes the principal eddy.

ageostrophic geopotential fluxes ${ }^{2}$ directed across the upper-level ridge directly downstream of the principal eddy and converging into the downstream cyclone, suggesting downstream development as the mechanism for vorticity generation in this eddy (Simmons and Hoskins 1979; Orlanski and Sheldon 1993). This is clarified in Fig. 7, which shows these fluxes in more detail and depicts a very strong southeastward geopotential flux that converges into the downstream eddy. The development due to ageostrophic flux convergence is enhanced by baroclinic development as indicated by the positive heat fluxes due to sinking cold air, shown in Fig. 4c.

The source of these upper-level geopotential fluxes is clearly the principal eddy. The baroclinic development of the principal eddy is converted into kinetic energy in the downstream eddy via geopotential fluxes at upper levels. If significant low-level baroclinicity is present, the downstream eddy will fully develop into its own baroclinic eddy. However, when this baroclinicity is absent or severely depleted, as is the case here because of continuous erosion by the eddies, ageostrophic fluxes from the downstream eddy converge upstream into the principal eddy at the surface, leaving the downstream eddy as an equivalent barotropic disturbance. This positive feedback results in the two eddies growing as a couplet. This description of a distinct mode of baroclinic development clearly cannot be captured by normal mode analysis. It should be noted that the strong equatorward direction of the ageostrophic fluxes is reminiscent of that found in barotropic waves emanating from a midlatitude source. On the sphere, these waves tend to propagate in the downstream and equatorward direction

\footnotetext{
${ }^{2}$ The ageostrophic geopotential flux is the only component of the total flux that has a divergent component and, so, is the only component that contributes to the eddy kinetic energy tendency (Orlanski and Sheldon 1993).
}

(Hoskins and Karoly 1981). Here, the downstream wave also behaves like an upper-level barotropic wave and will likely remain so unless it can tap into surface baroclinicity. This point will be addressed further below.

This energy cycle discussed in the previous section is illustrated in Fig. 8, which shows the downstream fluxes at upper levels and upstream fluxes at lower levels. There is strong downstream convergence of these fluxes at upper levels and upstream convergence at lower levels, although in the surface anticyclone there is also a strong cyclonic component to these fluxes that does not contribute to its convergence. The vertically integrated flux is downstream.

The interaction between the upstream and downstream eddies in forming the couplet can be clearly seen by examining the maximum amplitude of the upper- and lower-level vorticity in these eddies as a function of the lag, as shown in Fig. 9. It is strikingly apparent that the upstream eddy has the largest surface vorticity, and the downstream eddy has the largest upper-level vorticity, over the entire life cycle of the couplet. Further, the growth pattern in the vorticity shown in Fig. 9 is consistent with the description presented above of couplet development in terms of ageostrophic geopotential fluxes.

The couplet described here is clearly the principal feature in the regressed fields. However, there are often active eddies evolving both upstream and downstream of a couplet, but these eddies seem to be uncorrected in the long-term regression. Nevertheless, the characteristic structure of the couplet can be identified in specific instances in the storm track simulation. For example, a Hovmoeller diagram of the upper-level geopotential over a 15-day period is shown in Fig. 10, in which at least two trough-ridge-trough systems can be identified. The instantaneous three-dimensional structure of the couplet at day 293 of the storm track simulation is shown in Fig. 11. The similarity between the principal eddies, the downstream vorticity columns, and 


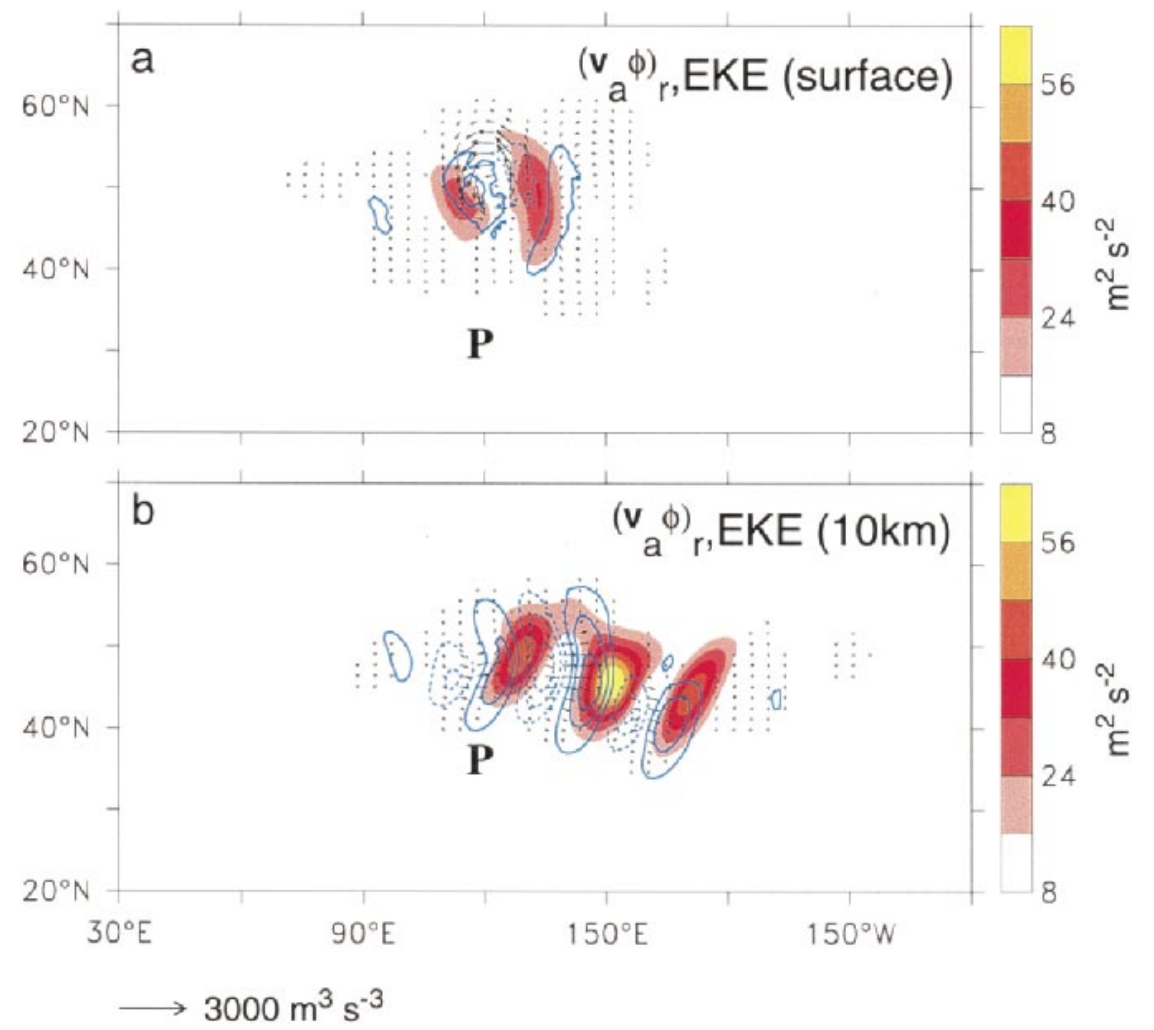

FIG. 8. Regressed eddy kinetic energy (color shading in $\mathrm{m}^{2} \mathrm{~s}^{-2}$ ), ageostrophic geopotential flux vectors, and ageostrophic geopotential flux convergence (contoured every $1 \times 10^{-3} \mathrm{~m}^{2} \mathrm{~s}^{-3}$ ) at (a) the surface and (b) $10 \mathrm{~km}$. Note that the fluxes are scaled by one-third compared to those shown in Fig. 7. The longest flux vector corresponds to about $1000 \mathrm{~m}^{3} \mathrm{~s}^{-3}$.

the upper-level geopotential fluxes in the regressed fields shown in Fig. 3 and instantaneous fields shown in Fig. 11 is remarkable and corroborates the description of the couplet provided above.

The identification of a couplet structure, consisting

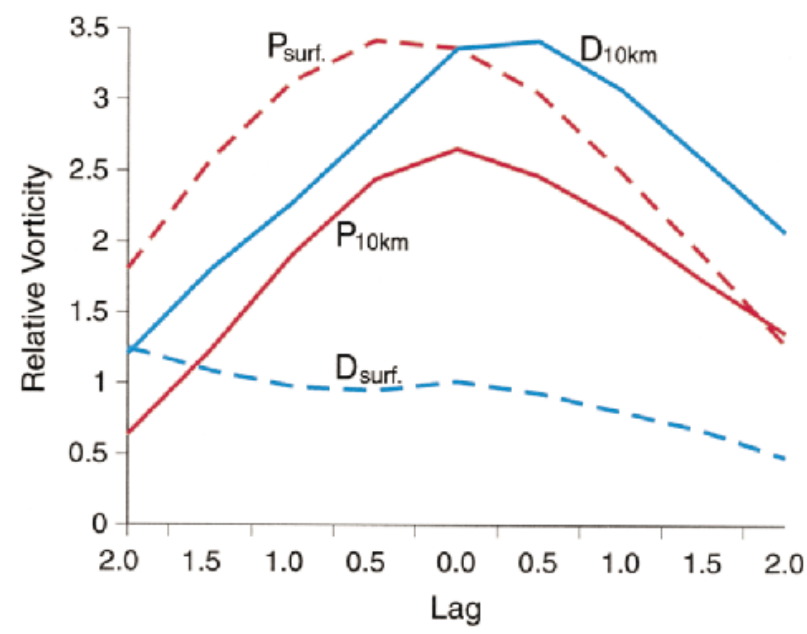

FIG. 9. Maximum regressed relative vorticity as a function of lag for the principal eddy (red curves) and downstream eddy (blue curves) at upper levels (solid) and the surface (dashed). of a ridge surrounded by two troughs, is one of the principal results of this analysis. As shown in Fig. 3, the western trough in the couplet is maintained by lowlevel baroclinicity (Fig. 4b) and the downstream trough by ageostrophic geopotential flux convergence. This couplet structure can be compared to what synopticians refer to as a building ridge, which in the present simulation moves at the baroclinic phase speed. One can conjecture that the couplet structure could act as a seed for an "omega" block, in the following way. If these systems are intense enough and have depleted the lowlevel baroclinicity, and the structure is mostly barotropic, the upstream lower-level fluxes can compensate the upper-level downstream fluxes, producing zero net energy dispersion from the system, thereby creating a very robust, long-lived feature. This issue will be addressed further in a subsequent paper.

Lee and Held (1993) have discussed the formation of baroclinic wave packets within zonally symmetric storm tracks. In these packets, the upstream eddy fluxes energy into the downstream eddy, which becomes large only when the upstream eddy starts to decay. However, in the couplet, the upstream and downstream eddies grow simultaneously, and indeed the downstream eddy is stronger in this case throughout the growth of the system. This characteristic is strikingly clear in individual 


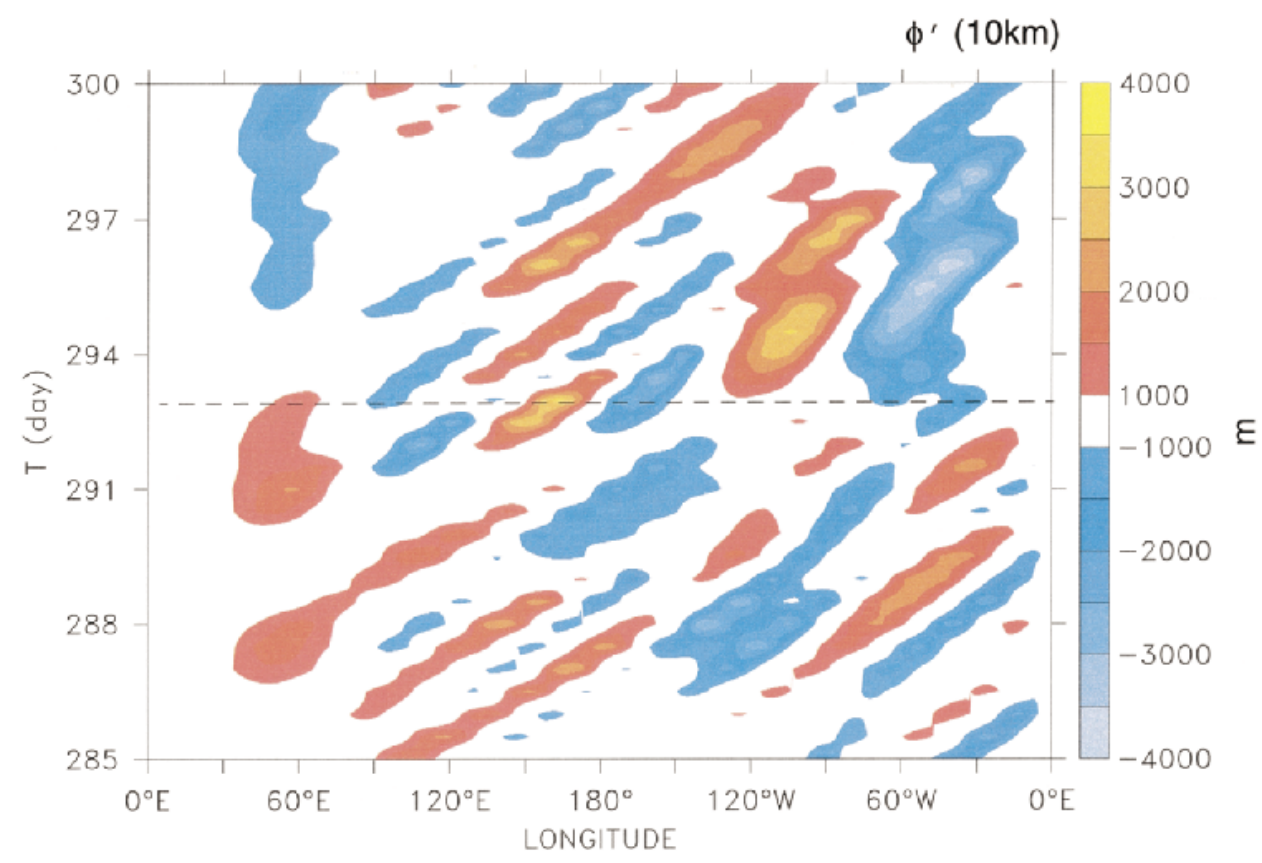

FIG. 10. Hovmoeller diagram of the perturbation geostrophic streamfunction at $10 \mathrm{~km}$ (color shading in $\mathrm{m}^{2} \mathrm{~s}^{-2}$ ) meridionally averaged over the latitude band $40^{\circ}-55^{\circ} \mathrm{N}$, for a 15 -day period in the storm track simulation. The dashed line at day 293 corresponds to the time shown in Fig. 11.

events, in which, for example, the surface vorticity in the couplet is larger in the upstream wave at all times, whereas the upper-level vorticity is largest in the downstream wave. This also is clear even in the regression (Fig. 3), which would tend to diminish the amplitude of the downstream eddy as it is farther from the regression point. Moreover, these results suggest that there is a distinct difference between a regime that supports wave packets and one that does not. Since the upperlevel downstream eddy tends to propagate equatorward in the absence of significant surface baroclinicity, it will suffer strong stretching as it reaches the subtropical critical layer. This will prevent the formation of wave packets. However, a supply of surface baroclinicity along the path of the storm track will inhibit meridional migration of the eddies and may promote baroclinic development and subsequent formation of wave packets.

\section{Eddy decay in the storm track}

The three-dimensional depictions of the regressed vorticity and potential temperature shown in Fig. 3. demonstrate that as the cyclonic vorticity intensifies, the potential temperature wraps around the vortex as the mean baroclinicity is diminished when warm air is advected northward ahead of the eddy and cold air is advected southward behind it. However, the regression is a weighted average, and the description of the eddy evolution should be verified in individual events that compose the regressed fields. A time sequence of the instantaneous low-level potential temperature for a pe- riod of 9 days is shown in Fig. 12. The intense roll-up of individual eddies is obvious, as is the strong poleward movement of the cyclonic centers. The characteristics of the flow that promote this intense roll-up are considered here.

The surface potential temperature is governed by

$$
\frac{\partial \theta}{\partial t}=-\frac{u}{a \cos \varphi} \frac{\partial \theta}{\partial \lambda}-\frac{v}{a} \frac{\partial \theta}{\partial \varphi}
$$

in the absence of dissipation. It is easy to see that where the meridional gradient of potential temperature vanishes, zonal potential temperature advection must account for the movement of the system at the wave phase speed $C=U_{0} ; \partial \theta / \partial t$, which is essentially due to movement of the system at the wave phase speed $C=U_{0}$, must also be equal to

$$
\frac{\partial \theta}{\partial t}=-\frac{U_{0}}{a \cos \varphi} \frac{\partial \theta}{\partial \lambda}=-\frac{u}{a \cos \varphi} \frac{\partial \theta}{\partial \lambda},
$$

so that at that point, the particle velocity must equal the phase velocity of the wave. A similar argument was considered by Orlanski and Bryan (1969) when interpreting the vertical breaking of gravity waves. That this is the case is shown by the coincidence of the $25 \mathrm{~m} \mathrm{~s}^{-1}$ contour of zonal velocity $\left(u=U_{0}\right.$ ) and the point in the low-level potential temperature field where the meridional gradient vanishes in Fig. 13. The signature of the individual critical regions is clearly seen in the timemean flow as well, and indeed the regressed vorticity centers approximately follow the $25 \mathrm{~m} \mathrm{~s}^{-1}$ time-mean 


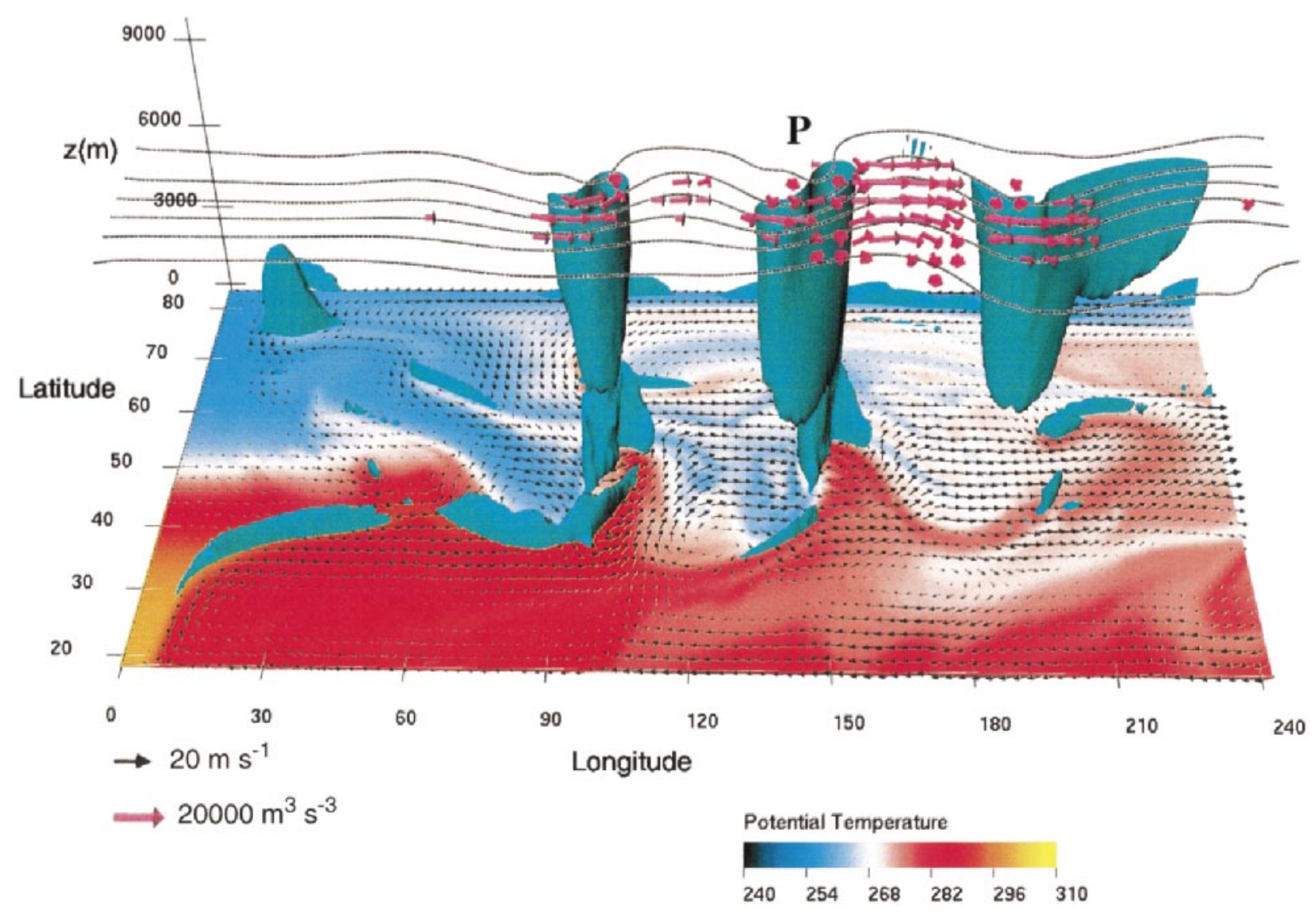

FIG. 11. As in Fig. 2, except the model variables at day 293 of the storm track simulation are shown. The value of the perturbation relative vorticity isosurface is $7 \times 10^{-5} \mathrm{~s}^{-1}$.

zonal velocity contour $\left(\bar{u} \approx U_{0}\right)$, as seen in Fig. $13 \mathrm{a}$. It is worth noting that linear theories of critical layers emphasize the time-mean flow characteristics, but these characteristics actually reflect the persistent collocation of local critical regions with temperature roll-up in individual eddies.

The surface vortex moves poleward and westward relative to the maximum temperature anomaly, a common feature of mature baroclinic eddies. This behavior can be explained by noting that at low levels the vorticity, as distinct from the potential temperature anomaly, requires stretching for it to propagate at the phase speed of the baroclinic system because the particle velocity at the vorticity center is smaller than the phase speed. The poleward movement is simply due to heat fluxes that move the region of stretching poleward. However, the westward displacement of the vorticity center occurs only as the vortex matures. At this stage, the potential temperature will commence rolling up, thereby shrinking the baroclinic forcing by reducing the source of stretching. As a consequence, the speed of the vortex center will be reduced from the phase speed and will be displaced slightly westward relative to the potential temperature anomaly. It is clear that the particle velocity will saturate at speeds close to the phase velocity south of the cyclonic vorticity centers.

When the low-level temperature rolls up, the baroclinicity is depleted, thereby preventing any further growth of the surface vorticity. As a consequence, the upper-level vorticity disconnects from the surface fields. However, the upper-level disturbance may still be characterized as an edge wave with an associated potential vorticity (PV) anomaly, which continues to propagate at the phase speed $U_{0}$. Depending on how much the wave radiates equatorward, it may reach upper-level critical layers where it can develop zero meridional potential vorticity gradients on isentropic surfaces. Since the jet at upper levels is stronger than $U_{0}$, it is expected that the isotachs of $u=U_{0}$ will bound the storm track over its entire length. Where the potential vorticity contours cross this isotach, it is expected that $\partial \mathrm{PV} / \partial y=0$ or the meridional velocity $v$ is zero. An example of this can be seen in the time sequence of upper-level PV shown in Fig 14. The PV at $320 \mathrm{~K}$ is shown at the times corresponding to those shown in Fig. 12, and there is a remarkable correspondence between points where $\partial \mathrm{PV} / \partial y=0$ and isotachs corresponding to the wave phase speed. 


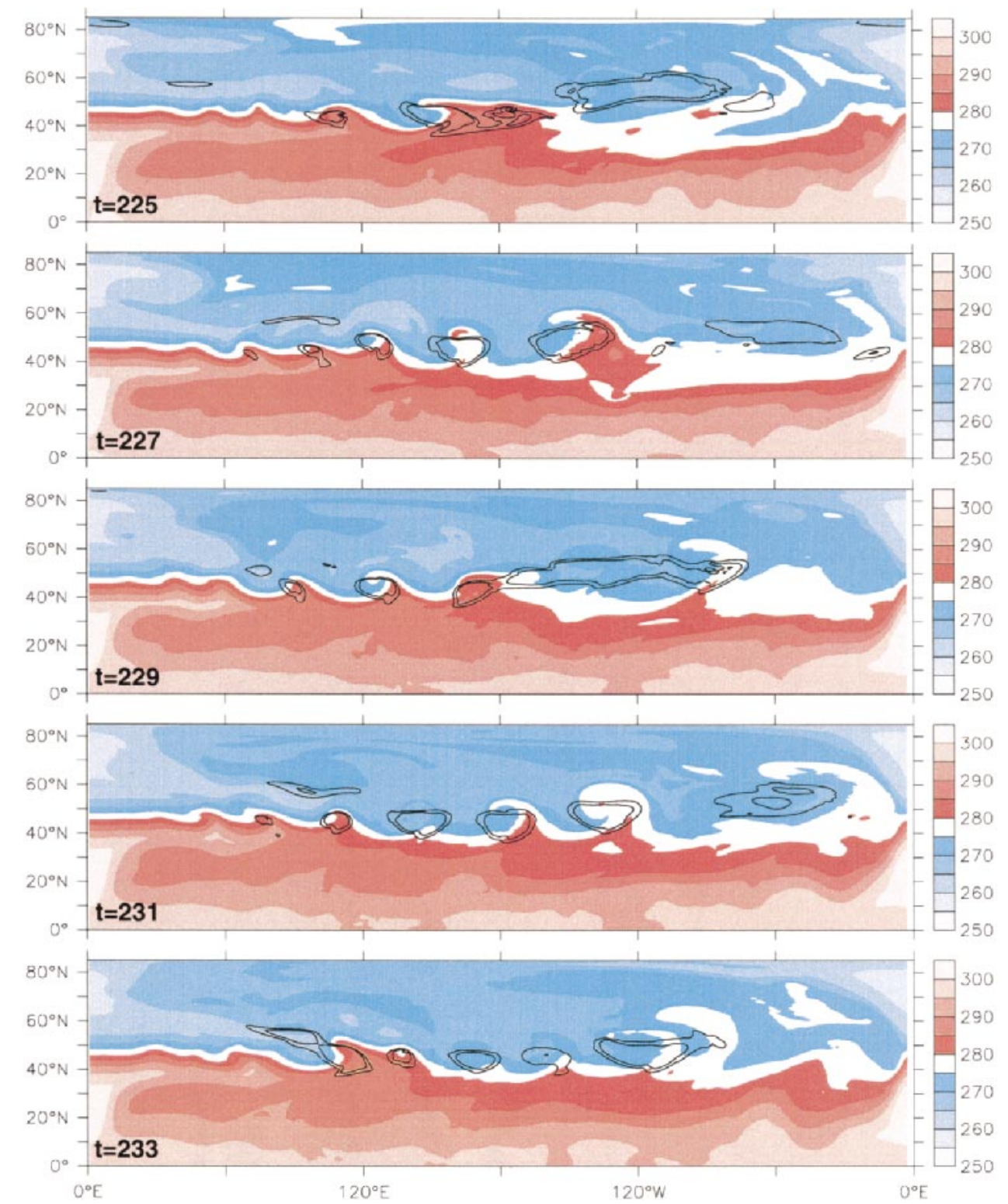

FIG. 12. Instantaneous depictions of the surface potential temperature (color shading in $\mathrm{K}$ ) and zonal wind (25 and $30 \mathrm{~m} \mathrm{~s}^{-1}$ contours) at (a) $t=225$, (b) $t=227$, (c) $t=229$, (d) $t=231$, and (e) $t=233$. The entire model domain is shown.

According to the previous discussion, it follows that since vortex roll-up at lower levels occurs when the zonal wind is close to the phase speed of the baroclinic eddies, the time-mean zonal wind at the lower levels will be closely related to the phase speed of these eddies. However, the baroclinic phase speed $U_{0}$ could be in principle determined by upstream conditions using a simple linear analysis of the stability of the basic-state jet. Usually, this phase velocity corresponds to the meridional average of the jet at the so-called steering height. In this sense, upstream conditions could provide a powerful direct predictor of the lower-level downstream conditions, because the maximum surface wind in the middle of the storm track will closely correspond to the phase speed of the eddies so determined. However, it should be pointed out that in different normalmode studies (e.g., Simmons and Hoskins 1978, 1980; Balasubramanian and Garner 1997a,b) the low-level velocity attained in these simulations is much larger than the normal-mode phase velocity (about twice as much). In fact, Simmons and Hoskins (1978) showed that the maximum velocity is attained very abruptly when the deep normal mode collapses and and becomes more zonal, at which point most of the accumulated eddy energy in the normal mode is deposited into the zonal flow. This is a very unrealistic feature of the decay of 


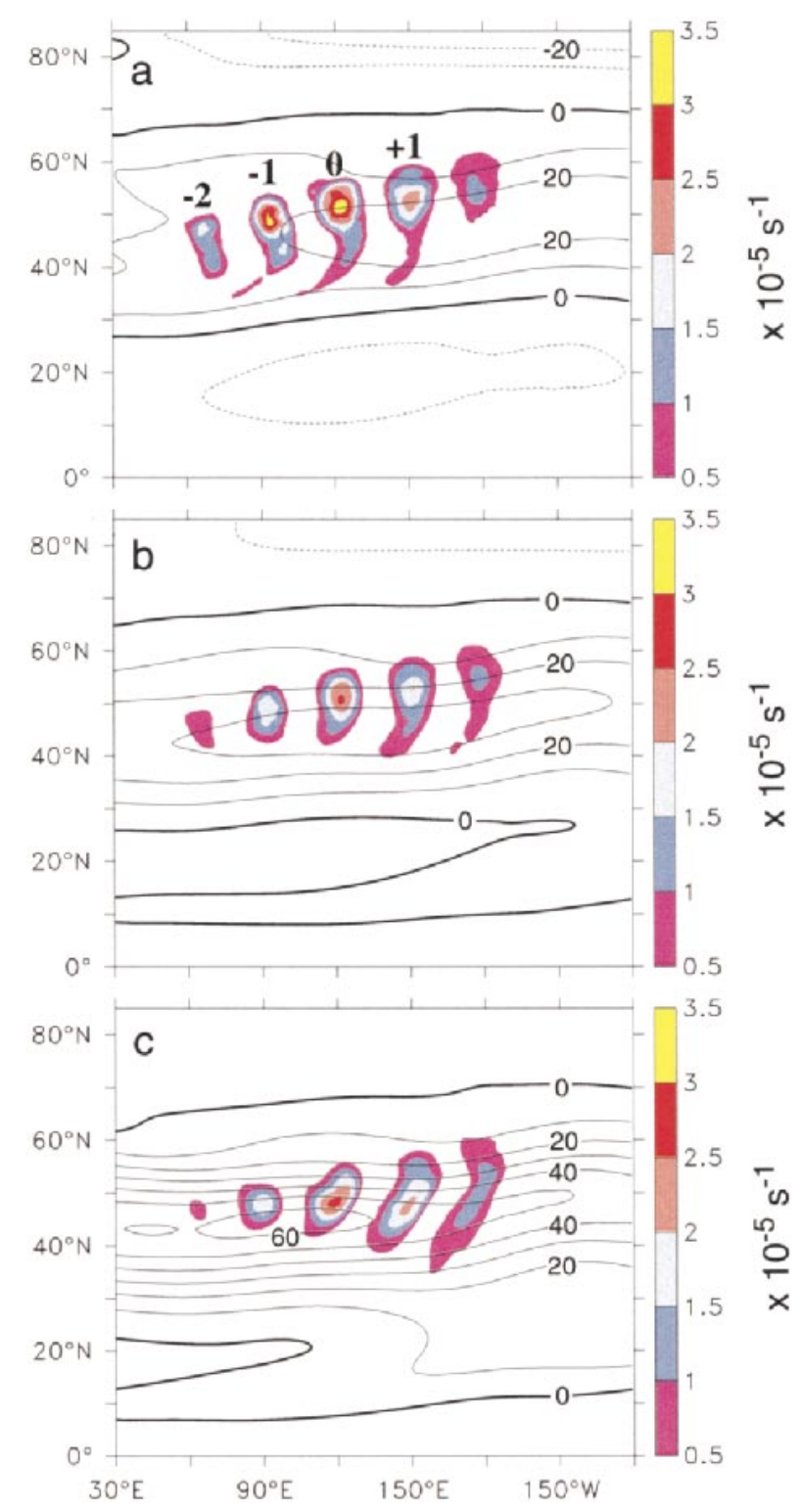

FIG. 13. The regressed cyclonic relative vorticity (color shading in $10^{-5} \mathrm{~s}^{-1}$ ) corresponding to the indicated time lags and the mean zonal wind (contoured every $10 \mathrm{~m} \mathrm{~s}^{-1}$; zero contour heavy, negative values dashed) at (a) the surface, (b) $4 \mathrm{~m}$, and (c) $10 \mathrm{~km}$.

normal modes. The ability of baroclinic eddies in a storm track environment to decay by fluxing energy downstream produces barotropic upper-level eddies that can propagate eastward and equatorward as a neutral barotropic wave where they decay by shredding into quasi-stationary zonal anomalies. Here, this decay produces a low-level zonal wind that corresponds quite closely to the baroclinic phase speed, as shown in Fig. 15 , in which the isotach of $U_{0}$ extends to the surface and is nearly barotropic on the poleward side of the jet (cf. Fig. 4).

On the equatorward side of the jet, horizontal shear will shred the PV and the wave will strongly dissipate, depending on how far equatorward the wave has progressed. At lower levels, the beginning of the roll-up is highly nonlinear because it is only late in the development that particle velocities equal the phase speed. However, at upper levels, there are always regions where the zonal wind corresponds to the wave phase speed. It is suggested that the equatorward distance the waves propagate is strongly related to the distance between these contours. For example, in a narrow jet, waves will be constrained to propagate pretty much zonally and lose small amounts of energy at the critical levels on both sides. A wider upper-level jet relative to the eddy size may allow greater equatorward excursions of the disturbance centers until they reach the critical layer, thereby breaking more anticyclonically, and storm track termination is more abrupt. An intermediate case is presented here.

The high-frequency vorticity forcing shown in Fig. 1 can perhaps be explained by these characteristics of the life cycle. Cyclonic vorticity forcing on the poleward side of the western half of the storm track is due to lowlevel cyclonic wave breaking, and conversely, the equatorward downstream anticyclonic forcing is due to the upper-level anticyclonic breaking of the eddies.

\section{Summary and conclusions}

The life cycle of baroclinic eddies in a controlled storm track environment has been examined by means of long integrations on a hemisphere. A time-lagged regression that captures disturbances with large meridional velocities has been applied to the meteorological variables. This regressed solution was used to describe the life cycle of the baroclinic eddies. A remarkable picture has emerged from this analysis detailing the growth, maturation, and decay of the eddies within a storm track.

The eddies grow as expected by strong poleward heat fluxes at low levels in regions of strong surface baroclinicity at the entrance of the storm track, in a manner similar to that of Charney modes. As the eddies evolve into a nonlinear regime, they penetrate vertically by fluxing energy upward, and the characteristic westward tilt exhibited in the vorticity vanishes by rotating into a meridional tilt, in which the lower-level cyclonic vorticity center moves poleward and the upper-level center moves equatorward. The poleward movement of the low-level vorticity is accomplished by the flux of timemean absolute vorticity as well as nonlinear vorticity fluxes. However, the north-south elongation of the upper-level vorticity is achieved by the extension of the surface vorticity into upper levels, combined with nonlinear effects that rotate the vorticity equatorward.

The classic picture of baroclinic evolution can be radically modified by the simultaneous development of an upper-level eddy downstream of the principal eddy. The results presented here show that this eddy is an integral part of a self-sustained system, here named as a couplet, 


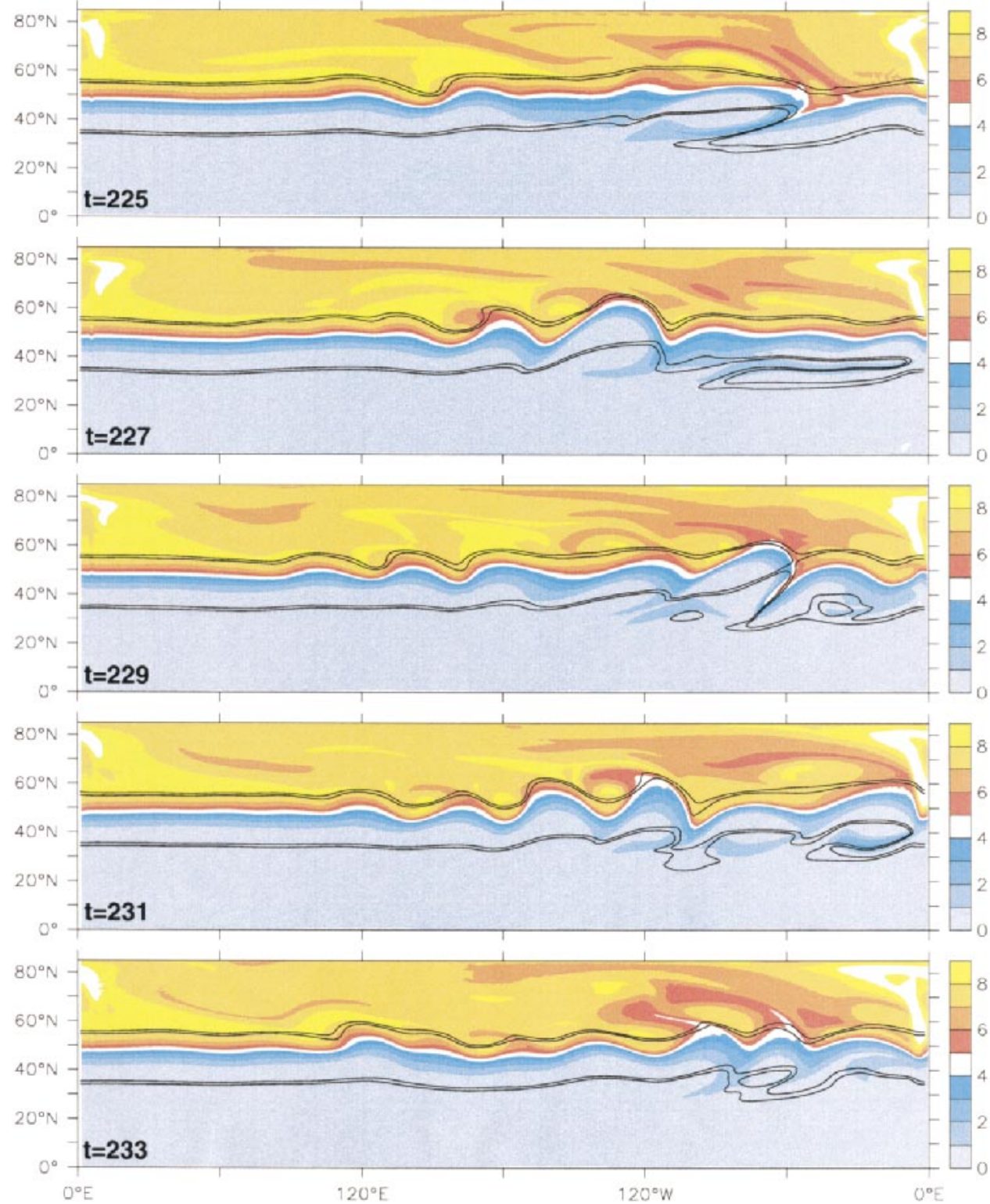

FIG. 14. Instantaneous depictions of the potential vorticity (color shading in PVU $=10^{-6} \mathrm{~m}^{2} \mathrm{~s}^{-1} \mathrm{~K} \mathrm{~kg}^{-1}$ ) and zonal wind ( 25 and $30 \mathrm{~m} \mathrm{~s}^{-1}$ contours) on the $320 \mathrm{~K}$ surface at the times corresponding to the panels shown in Fig. 13

such that the upstream principal eddy fluxes energy to the upper-level downstream eddy, whereas at lower levels the principal eddy receives energy fluxes from its downstream companion but grows primarily from baroclinic sources. The signature of the couplet at upper levels will resemble an omega-like pattern with a trough in the west (principal eddy) and one to the east (downstream eddy) separated by a developing ridge, well known by synopticians as a "building ridge." This structure is critically dependent on the strong zonal gradients in baroclinicity encountered within the storm track environment. It could be conjectured that a regime that produces couplets cannot produce wave packets.

A second important result revealed by this analysis is the fact that when low-level vorticity centers migrate poleward, they tend to follow isotachs that closely correspond to the phase velocity of the eddies. It has been shown that as the low-level vorticity is enhanced by baroclinic processes, it reaches a stage where particle velocities south of the center exceed the phase speed, at which point the potential temperature gradient reverses. This is very nicely illustrated in a time sequence 


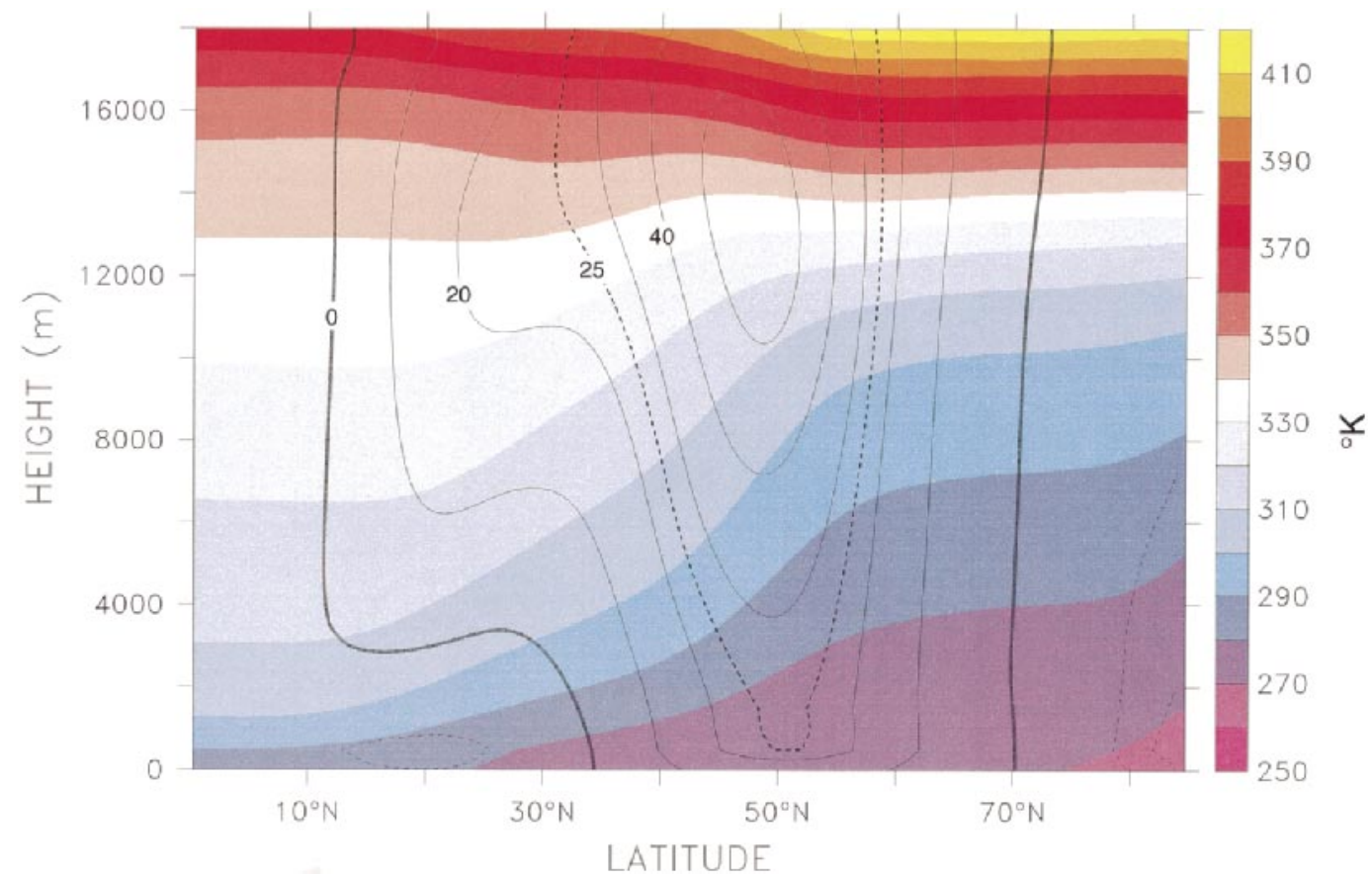

FIG. 15. Time-mean zonal wind (contoured every $10 \mathrm{~m} \mathrm{~s}^{-1}$; zero contour heavy, negative values dashed) and potential temperature (color shading in K), zonally averaged from $180^{\circ}$ to $270^{\circ} \mathrm{E}$ (the third quarter of the channel). The heavy dashed contour corresponds to a time-mean zonal wind of $U_{0} \approx 25 \mathrm{~m} \mathrm{~s}^{-1}$, the approximate phase speed of the eddies in the storm track.

of the surface potential temperature. It is suggested that the maximum westward momentum that the eddies deposit at lower levels must closely correspond to the phase velocity, which can actually be estimated from just the upstream conditions when the eddies are small. Similar arguments can be applied to the potential vorticity at upper levels, although the eddies here tend to behave more linearly than at lower levels because the zonal flow is much stronger and the particle velocities are close to the phase speed only near the meridional boundaries of the storm track. The intensity and direction of propagation of these waves will determine the overall structure of the storm track. Equatorward propagation that reaches critical layers more quickly will result in wave breaking that produces an early termination of the storm track. The upper-level critical layers thus tend to act as a waveguide. It is possible that the termination of the storm track could depend on the width of the jet at the storm track entrance-wider upper-level jets allow more equatorward eddy propagation with more intense wave breaking and an early termination as noted above.

There are a substantial number of issues that are raised by this analysis. Among them are the magnitude of the mean surface speed and its relation to the propagation speed of the eddies, the dependence of the storm track length on the width of the upstream jet, how the structure of the couplet influences the development of long-lived features within the storm track, and the sensitivity of storm track structure to the width of the jet; all of these aspects will be considered in a future investigation.

Acknowledgments. The authors would like to thank Drs. Steve Garner, Isaac Held, Paul Kushnir, and Jerry Mahlman for providing comments that greatly clarified this presentation. The authors also extend their appreciation to Dr. Brian Hoskins and an anonymous reviewer for suggestions that helped further clarify the article, and to Cathy Raphael for her assistance in producing the figures.

\section{REFERENCES}

Balasubramanian, G., and S. T. Garner, 1997a: The role of momentum fluxes in shaping the life cycle of a baroclinic wave. J. Atmos. Sci., 54, 510-533.

— J. Atmos. Sci., 54, 2850-2871.

Chang, E. K. M., 1993: Downstream development of baroclinic waves as inferred from regression analysis. J. Atmos. Sci., 50, 2038 2053.

_ , and I. Orlanski, 1993: On the dynamics of a storm track. $J$. Atmos. Sci., 50, 999-1015.

Haltiner, G. J., and R. T. Williams, 1980: Numerical Prediction and Dynamic Meteorology. 2d ed. John Wiley and Sons, 477 pp.

Hoskins, B. J., 1976: Baroclinic waves and frontogenesis, Part I. Quart. J. Roy. Meteor. Soc., 102, 103-122.

- 1983: Modelling of the transient eddies and their feedback on the mean flow. Large-Scale Dynamical Processes, B. J. Hoskins and R. P. Pearce, Eds., Academic Press, 169-199.

, and N. V. West, 1979: Baroclinic waves and frontogenesis. Part 
II: Uniform potential vorticity jet flows-Cold and warm fronts. J. Atmos. Sci., 36, 1663-1680.

— and D. J. Karoly, 1981: The steady linear response of a spherical atmosphere to thermal and orographic forcing. J. Atmos. Sci. 38, 1179-1196

— , and P. J. Valdes, 1990: On the existence of storm-tracks. $J$. Atmos. Sci., 47, 1854-1864.

— I. N. James, and G. H. White, 1983: The shape, propagation and mean-flow interaction of large-scale weather systems. $J$. Atmos. Sci., 40, 1595-1612.

Illari, L., and J. C. Marshall, 1983: On the interpretation of eddy fluxes during a blocking episode. J. Atmos. Sci., 40, 2232-2242.

Lee, S., and I. M. Held, 1993: Baroclinic wave packets in models and observations. J. Atmos. Sci., 50, 1413-1428.

Lim, G. H., and J. M. Wallace, 1991: Structure and evolution of baroclinic waves as inferred from regression analysis. J. Atmos. Sci., 48, 1718-1732.

Nakamura, H., M. Nakamura, and J. L. Anderson, 1997: The role of high- and low-frequency dynamics in blocking formation. Mon Wea. Rev., 125, 2074-2093.
Orlanski, I., 1998: Poleward deflection of storm tracks. J. Atmos. Sci., 55, 2577-2602.

- , and K. Bryan, 1969: Formation of the thermocline step structure by large amplitude internal gravity wave. J. Geophys. Res., 74, 6975-6983

— velopment over western North America. Mon. Wea. Rev., 121, 2929-2950.

— development. J. Atmos. Sci., 51, 589-611.

Simmons, A. J., and B. J. Hoskins, 1978: The life cycles of some nonlinear baroclinic waves. J. Atmos. Sci., 35, 414-432.

, and - 1979: The downstream and upstream development of unstable baroclinic waves. J. Atmos. Sci., 36, 1239-1254.

, and - 1980: Barotropic influences on the growth and decay of nonlinear baroclinic waves. J. Atmos. Sci., 37, 1679-1684.

Wallace, J. M., G. H. Lim, and M. L. Blackmon, 1988: Relationship between cyclone tracks, anticyclone tracks, and baroclinic waveguides. J. Atmos. Sci., 45, 439-462.

Whitaker, J. S., and C. Snyder, 1993: The effects of spherical geometry on the evolution of baroclinic waves. J. Atmos. Sci., 50, 597-612. 